International Mathematical Forum, Vol. 8, 2013, no. 9, 427 - 432

HIKARI Ltd, www.m-hikari.com

\title{
An Abundancy Result for the Two Prime Power Case and Results for an Equations of Goormaghtigh
}

\author{
Richard F. Ryan \\ Marymount College \\ Rancho Palos Verdes, CA 90275-6299 USA \\ rryan@marymountpv.edu
}

Copyright (C) 2013 Richard F. Ryan. This is an open access article distributed under the Creative Commons Attribution License, which permits unrestricted use, distribution, and reproduction in any medium, provided the original work is properly cited.

\begin{abstract}
Let $a$ represent a positive integer, and $\sigma(a)$ denote the sum of the (positive) factors of $a$. The abundancy (or abundancy index) of $a$, denoted by $I(a)$, is defined by $I(a)=\sigma(a) / a$. Suppose that we are given positive integers $m_{0}$ and $n_{0}$ and distinct prime numbers $p$ and $q$. In the current note, new results concerning an equation of Goormaghtigh are established and used to show that the equation $I(x)=I\left(p^{m_{0}} q^{n_{0}}\right)$ has, at most, one solution of the form $x=p^{m} q^{n}$ such that $p^{m} q^{n} \neq p^{m_{0}} q^{n_{0}}$.
\end{abstract}

Mathematics Subject Classification: Primary: 11A25, Secondary: 11D61

Keywords: Abundancy, Goormaghtigh equation

\section{Introduction}

We will start with a review of some basic properties that is similar, at times, to the introductory section in [4]. If $p$ is a prime number and $m$ is a nonnegative integer, then the sum of the factors of $p^{m}$ is given by

$$
\sigma\left(p^{m}\right)=\sum_{j=0}^{m} p^{j}=\frac{p^{m+1}-1}{p-1} .
$$

Additionally, if $a=\prod_{j=1}^{k} p_{j}^{m_{j}}$, where $p_{1}, p_{2}, \ldots, p_{k}$ are distinct primes, $k$ is a positive integer, and $m_{1}, m_{2}, \ldots, m_{k}$ are nonnegative integers, then

$$
\sigma(a)=\prod_{j=1}^{k} \frac{p_{j}^{m_{j}+1}-1}{p_{j}-1} .
$$


Consequently, $\sigma$ is a multiplicative function; that is, if $a$ and $b$ are relatively prime then $\sigma(a b)=\sigma(a) \sigma(b)$. If we continue to express the positive integer $a$ in the form $\prod_{j=1}^{k} p_{j}^{m_{j}}$, then the abundancy of $a$ is given by

$$
I(a)=\frac{\sigma(a)}{a}=\prod_{j=1}^{k} \frac{p_{j}^{m_{j}+1}-1}{p_{j}^{m_{j}}\left(p_{j}-1\right)},
$$

where $I$ is multiplicative.

It is not difficult to show that, if $a$ is relatively prime to $\sigma(a)$, then $x=a$ is the unique solution of $I(x)=I(a)$. Suppose we are given a prime number $p$ and positive integer $m_{0}$. Since $p$ does not divide $\sigma\left(p^{m_{0}}\right)$, the only solution of $I(x)=I\left(p^{m_{0}}\right)$ is $x=p^{m_{0}}$. The equation

$$
I(x)=I\left(p^{m_{0}} q^{n_{0}}\right)
$$

has been studied by the current author [4], [5], [6]. When considering this equation, we will assume that $p$ and $q$ represent distinct prime numbers, $m_{0}$ and $n_{0}$ represent positive integers, and the values of $p, q$, and $m_{0}$ are given. We will assume that the value of $n_{0}$ is given too, except in the last paragraph of this article. Also, $Z^{+}$will denote the set of positive integers. Obviously, if $\sigma\left(p^{m_{0}} q^{n_{0}}\right)$ is relatively prime to $p q$, then $x=p^{m_{0}} q^{n_{0}}$ is the unique solution of (1). If $\operatorname{gcd}\left(\sigma\left(p^{m_{0}} q^{n_{0}}\right), p q\right)>1$, other solutions exist in some instances. When they exist, each additional solution of (1) can be written in the form

$$
x=p^{m} q^{n} d \text { such that }(m, n) \neq\left(m_{0}, n_{0}\right), d \in Z^{+}, \operatorname{and} \operatorname{gcd}(d, p q)=1 .
$$

If a solution of (1) is written in the form of (2), then $m \neq m_{0}$; likewise $n \neq n_{0}$. In (2), $m$ and $n$ represent non-negative integers.

There is some interest in solutions of (1) that can be expressed in the form

$$
x=p^{m} q^{n} \text { such that }(m, n) \neq\left(m_{0}, n_{0}\right) .
$$

Since we are assuming that $m_{0}, n_{0} \in Z^{+}$, it follows that the (integer) values of $m$ and $n$ are positive. (For if (3) is a solution of (1) such that $m=0$, then $I\left(q^{n}\right)=I\left(p^{m_{0}} q^{n_{0}}\right)$, which implies that $m_{0}=0$, contradicting the assumption that $m_{0}$ is positive. Similarly, $n \neq 0$.) The investigation concerning solutions in the form of (3) is related to the study of another well-known equation. An equation of Goormaghtigh is given by

$$
\frac{y^{u}-1}{y-1}=\frac{z^{w}-1}{z-1} \text { in integers } y>1, z>1, u>2, w>2 \text {, with } y<z .
$$

R. Ratat [3] and R. Goormaghtigh [1] noted that solutions to (4) are given by $(y, z, u, w)=(2,5,5,3)$ or $(2,90,13,3)$; it has been conjectured that these are the only solutions. B. He and A. Togbé [2] considered the equation

$$
\frac{y^{u}-1}{y-1}=\frac{z^{w}-1}{z-1} \text { in integers } z>y>1, u>1, w>1
$$

and established the following result. 
Theorem 1.1. (He and Togbé) Let $z>y>1$ be given integers. Then equation (5) has at most one solution $(u, w)$.

Let us recall another bit of common notation. If $c$ is a nonzero integer, then $\nu_{2}(c)$ is the nonnegative integer with the property that $2^{\nu_{2}(c)}$ divides $c$ but $2^{\nu_{2}(c)+1}$ does not divide $c$. With the aid of theorem 1.1, the following theorem was proved [4], [5].

Theorem 1.2. Suppose that

$$
I\left(p^{m_{1}} q^{n_{1}}\right)=I\left(p^{m_{2}} q^{n_{2}}\right)
$$

where $p$ and $q$ represent prime numbers, $m_{1}, n_{1}, m_{2}$, and $n_{2}$ are positive integers, and $p^{m_{1}} q^{n_{1}} \neq p^{m_{2}} q^{n_{2}}$; thus $p \neq q$. Without loss of generality, we will assume that $m_{1}<m_{2}$ and $p^{m_{2}-m_{1}}<q^{n_{1}-n_{2}}$ throughout this theorem. Let $t_{p}=\frac{m_{2}+1}{m_{2}-m_{1}}$ and $t_{q}=\frac{n_{1}+1}{n_{1}-n_{2}}$. Then:

(A) The greatest common divisor of $m_{1}+1$ and $m_{2}+1$ is $m_{2}-m_{1}$ and $\operatorname{gcd}\left(n_{1}+1, n_{2}+1\right)=n_{1}-n_{2}$.

(B) A solution of (4) is given by

$$
(y, z, u, w)=\left(p^{m_{2}-m_{1}}, q^{n_{1}-n_{2}}, t_{p}, t_{q}\right) .
$$

(C) It turns out that $m_{1}$ and $m_{2}$ are relatively prime; likewise for $n_{1}$ and $n_{2}$; additionally, $\nu_{2}\left(m_{1}+1\right) \neq \nu_{2}\left(m_{2}+1\right)$ and $\nu_{2}\left(n_{1}+1\right) \neq \nu_{2}\left(n_{2}+1\right)$.

The only known solution of (6) is $\left\{p^{m_{1}} q^{n_{1}}, p^{m_{2}} q^{n_{2}}\right\}=\{80,200\}$. Theorem 1.2 will be useful in the ensuing section.

\section{Main Results}

Even though the following theorem is not a major breakthrough in the study of equation (5), parts $\mathrm{A}$ and $\mathrm{B}$ are useful when establishing the primary result in this article.

Theorem 2.1. Suppose that $(y, z, u, w)=\left(a^{j_{1}}, b^{k_{1}}, u_{1}, w_{1}\right)$ is a solution of (5) such that $a, b, j_{1}$, and $k_{1}$ are positive integers, $a>1, b>1$, and $a$ is relatively prime to $b$.

(A) Let $j_{2}$ and $k_{2}$ represent positive integers such that $\frac{j_{1} u_{1}+j_{2}}{j_{2}}$ and $\frac{k_{1}\left(w_{1}-1\right)}{k_{2}}$ are integers greater than 1 . Then neither $\left(a^{j_{2}}, b^{k_{2}}, \frac{j_{1} u_{1}+j_{2}}{j_{2}}, \frac{k_{1}\left(w_{1}-1\right)}{k_{2}}\right)$ nor $\left(b^{k_{2}}, a^{j_{2}}, \frac{k_{1}\left(w_{1}-1\right)}{k_{2}}, \frac{j_{1} u_{1}+j_{2}}{j_{2}}\right)$ is a solution of $(5)$.

(B) Let $j_{3}$ and $k_{3}$ represent positive integers such that $\frac{j_{1}\left(u_{1}-1\right)}{j_{3}}$ and $\frac{k_{1} w_{1}+k_{3}}{k_{3}}$ are integers greater than 1 . Then neither $\left(a^{j_{3}}, b^{k_{3}}, \frac{j_{1}\left(u_{1}-1\right)}{j_{3}}, \frac{k_{1} w_{1}+k_{3}}{k_{3}}\right)$ nor $\left(b^{k_{3}}, a^{j_{3}}, \frac{k_{1} w_{1}+k_{3}}{k_{3}}, \frac{j_{1}\left(u_{1}-1\right)}{j_{3}}\right)$ is a solution of $(5)$. 
(C) Let $k_{4}$ and $w_{4}$ represent any two positive integers such that $w_{4}>1$. Then neither $\left(a^{j_{1}}, b^{k_{4}}, u_{1}-1, w_{4}\right)$ nor $\left(b^{k_{4}}, a^{j_{1}}, w_{4}, u_{1}-1\right)$ is a solution of $(5)$. Similarly, neither $\left(a^{j_{1}}, b^{k_{4}}, u_{1}+1, w_{4}\right)$ nor $\left(b^{k_{4}}, a^{j_{1}}, w_{4}, u_{1}+1\right)$ is a solution of $(5)$.

(D) Let $j_{4}$ and $u_{4}$ represent any two positive integers such that $u_{4}>1$. Then neither $\left(a^{j_{4}}, b^{k_{1}}, u_{4}, w_{1}-1\right)$ nor $\left(b^{k_{1}}, a^{j_{4}}, w_{1}-1, u_{4}\right)$ is a solution of $(5)$. Similarly, neither $\left(a^{j_{4}}, b^{k_{1}}, u_{4}, w_{1}+1\right)$ nor $\left(b^{k_{1}}, a^{j_{4}}, w_{1}+1, u_{4}\right)$ is a solution of (5).

Proof. (A) Suppose that $\left(a^{j_{1}}, b^{k_{1}}, u_{1}, w_{1}\right)$, and either $\left(a^{j_{2}}, b^{k_{2}}, \frac{j_{1} u_{1}+j_{2}}{j_{2}}, \frac{k_{1}\left(w_{1}-1\right)}{k_{2}}\right)$ or $\left(b^{k_{2}}, a^{j_{2}}, \frac{k_{1}\left(w_{1}-1\right)}{k_{2}}, \frac{j_{1} u_{1}+j_{2}}{j_{2}}\right)$, are solutions of (5). Then

$$
\frac{a^{j_{1} u_{1}}-1}{a^{j_{1}}-1}=\frac{b^{k_{1} w_{1}}-1}{b^{k_{1}}-1}
$$

and

$$
\frac{a^{j_{1} u_{1}+j_{2}}-1}{a^{j_{2}}-1}=\frac{b^{k_{1}\left(w_{1}-1\right)}-1}{b^{k_{2}}-1}
$$

Subtracting 1 from both sides of the equations in (8) and (9), we observe that

$$
a^{j_{1}} \frac{a^{j_{1}\left(u_{1}-1\right)}-1}{a^{j_{1}}-1}=b^{k_{1}} \frac{b^{k_{1}\left(w_{1}-1\right)}-1}{b^{k_{1}}-1}
$$

and

$$
a^{j_{2}} \frac{a^{j_{1} u_{1}}-1}{a^{j_{2}}-1}=b^{k_{2}} \frac{b^{k_{1}\left(w_{1}-1\right)-k_{2}}-1}{b^{k_{2}}-1} .
$$

Due to equations (8) and (11), $b^{k_{2}}$ divides $a^{j_{1}}-1$, and thus $b^{k_{2}}<a^{j_{1}}$. However, from equations (9) and (10), we see that $a^{j_{1}}$ divides $b^{k_{2}}-1$. Thus $a^{j_{1}}<b^{k_{2}}$, and we have a contradiction.

(B) The proof of part B is similar to the proof of part A of this theorem.

(C) Let us suppose that $\left(a^{j_{1}}, b^{k_{1}}, u_{1}, w_{1}\right)$, and either $\left(a^{j_{1}}, b^{k_{4}}, u_{1}-1, w_{4}\right)$ or $\left(b^{k_{4}}, a^{j_{1}}, w_{4}, u_{1}-1\right)$, are solutions of (5). Obviously, $u_{1}>2$ in this case and equation (10) continues to be true. Moreover,

$$
\frac{a^{j_{1}\left(u_{1}-1\right)}-1}{a^{j_{1}}-1}=\frac{b^{k_{4} w_{4}}-1}{b^{k_{4}}-1}
$$

In equation (10), $b^{k_{1}}$ divides $\frac{a^{j_{1}\left(u_{1}-1\right)}-1}{a^{j_{1}}-1}$. But, in equation (12), $b^{k_{1}}$ is relatively prime to the same fraction, and we have a contradiction. If, once again, $\left(a^{j_{1}}, b^{k_{1}}, u_{1}, w_{1}\right)$ satisfies $(5)$ and we assume that $\left(a^{j_{1}}, b^{k_{4}}, u_{1}+\right.$ 
$\left.1, w_{4}\right)$ or $\left(b^{k_{4}}, a^{j_{1}}, w_{4}, u_{1}+1\right)$ satisfies the same equation, then (8) remains true and

$$
a^{j_{1}} \frac{a^{j_{1} u_{1}}-1}{a^{j_{1}}-1}=b^{k_{4}} \frac{b^{k_{4}\left(w_{4}-1\right)}-1}{b^{k_{4}}-1} .
$$

Thus $b^{k_{4}}$ is relatively prime to $\frac{a^{j_{1} u_{1}}-1}{a^{j_{1}}-1}$ and a factor of the same fraction, which is an obvious contradiction.

(D) The proof of this result is similar to the proof of part C.

As you will see, the proof of the next result is easy to attain at this point. We continue to assume that, when considering equation (1), the values of the distinct primes $p$ and $q$, and the positive integers $m_{0}$ and $n_{0}$, are given.

Theorem 2.2. There is, at most, one solution of (1) in the form given by (3).

Proof. Suppose that $p^{m_{1}} q^{n_{1}}, p^{m_{2}} q^{n_{2}}$, and $p^{m_{3}} q^{n_{3}}$ are distinct solutions of (1). Without loss of generality, we will assume that $m_{1}<m_{2}<m_{3}$; thus $n_{1}>n_{2}>$ $n_{3}$. Furthermore, we will assume that $p^{m_{0}} q^{n_{0}}$ is equal to one of these solutions. Again, let $t_{p}=\frac{m_{2}+1}{m_{2}-m_{1}}$ and $t_{q}=\frac{n_{1}+1}{n_{1}-n_{2}}$. Due to theorem 1.2(B), either (7) or

$$
\left(q^{n_{1}-n_{2}}, p^{m_{2}-m_{1}}, t_{q}, t_{p}\right)
$$

is a solution of (4). For the moment, we will assume that (7) is a solution of (4), and thus, a solution of (5). Applying theorem 1.2(B) again, we see that

$$
\left(p^{m_{3}-m_{2}}, q^{n_{2}-n_{3}}, \frac{m_{3}+1}{m_{3}-m_{2}}, \frac{n_{2}+1}{n_{2}-n_{3}}\right)
$$

or

$$
\left(q^{n_{2}-n_{3}}, p^{m_{3}-m_{2}}, \frac{n_{2}+1}{n_{2}-n_{3}}, \frac{m_{3}+1}{m_{3}-m_{2}}\right)
$$

is a solution of (4) and (5). Let $j_{1}=m_{2}-m_{1}, k_{1}=n_{1}-n_{2}, j_{2}=m_{3}-m_{2}$, and $k_{2}=n_{2}-n_{3}$. Then (14) and (15) can be rewritten as $\left(p^{j_{2}}, q^{k_{2}}, \frac{j_{1} t_{p}+j_{2}}{j_{2}}, \frac{k_{1}\left(t_{q}-1\right)}{k_{2}}\right)$ and $\left(q^{k_{2}}, p^{j_{2}}, \frac{k_{1}\left(t_{q}-1\right)}{k_{2}}, \frac{j_{1} t_{p}+j_{2}}{j_{2}}\right)$ respectively, and we have a contradiction to theorem 2.1(A). We get a similar contradiction, to theorem 2.1(B), if we make the assumption that (13) is a solution of (4).

It was previously established [4] that, if $m_{0}$ and $n_{0}$ are even and the distinct primes $p$ and $q$ are odd, then there is no solution of (1) in the form of (3). In the same article it was shown that, if $m_{0}$ (or $n_{0}$ ) is equal to 1 , then $x=200$ is the only solution of (1) in the form of (3); this solution occurs when $p q^{n_{0}}$ (or respectively, $p^{m_{0}} q$ ) is equal to 80 . 
In this paragraph, we are assuming that the values of $p, q$, and $m_{0}$ are given. It has been demonstrated [4] that equation (1) has (at most) finitely many solutions $\left(x, n_{0}\right)$ such that $x$ is of the form specified in (3). It was also verified [4] that, if $m_{0}+1$ is an odd prime, then there are at most two values of $n_{0}$ that will yield a solution of (1) such that $x$ is of the form given by (3). Presently, we can improve on this statement. Due to parts $\mathrm{C}$ and D of theorem 2.1 , if $m_{0}+1$ is an odd prime, then there is at most one solution $\left(x, n_{0}\right)$ of (1) such that $x$ is of the form given in (3). The proof of this result may be obtained from the author. Lastly, if we apply theorem 2.1, parts C and D, in a similar fashion to example 3.3 from the article cited in this paragraph, we observe that, when $m_{0}=11$, there are at most four solutions $\left(x, n_{0}\right)$ of $(1)$ such that $x$ is of the form indicated in (3).

\section{References}

[1] R. Goormaghtigh, L'Intermédiaire des Mathématiciens, 24 (1917), 88.

[2] B. He and A. Togbé, On the number of solutions of Goormaghtigh equation for given $x$ and $y$, Indag. Math. (N.S.), 19 (2008), 65 - 72.

[3] R. Ratat, L'Intermédiaire des Mathématiciens, 23 (1916), 150.

[4] R. F. Ryan, Improvements on previous abundancy results, Int. J. Contemp. Math. Sci., 4 (2009), 1299 - 1313.

[5] R. F. Ryan, Results concerning an equation of Goormaghtigh and related topics, Int. Math. Forum, 1 (2006), 1195 - 1206.

[6] R. F. Ryan, Results concerning uniqueness for $\sigma(x) / x=\sigma\left(p^{n} q^{m}\right) /\left(p^{n} q^{m}\right)$ and related topics, Int. Math. J., 2 (2002), 497 - 514.

\section{Received: November, 2012}

